\title{
Study of HSA interactions with arachidonic acid using spectroscopic methods revealing molecular dynamics of HSA-AA interactions
}

\author{
FERESHTE MAHDIZADE VALOJERDI ${ }^{1}$, ALIREZA FARASAT ${ }^{2}$, \\ HANIFEH SHARIATIFAR $^{3}$ and NEMATOLLAH GHEIBI ${ }^{2}$
}

\author{
${ }^{1}$ Department of Biology, Sciences and Research Branch, Islamic Azad University, Tehran 8244865179; \\ ${ }^{2}$ Cellular and Molecular Research Center, Qazvin University of Medical Sciences, Qazvin 3419915315; \\ ${ }^{3}$ Cellular and Molecular Research Center, Research Institute for Health Development, \\ Kurdistan University of Medical Sciences, Sanandaj 6618634683, Iran
}

Received March 17, 2019; Accepted September 20, 2019

DOI: $10.3892 /$ br.2019.1270

\begin{abstract}
The interaction between human serum albumin (HSA) and arachidonic acid (AA) as an unsaturated fatty acid were investigated in the present study using methods including UV-VIS spectrophotometry, fluorescence and circular dichroism (CD) spectroscopy, lifetime measurements, fluorescence anisotropy measurements and visual molecular dynamics (MD). The thermodynamic parameters were assessed from HSA thermal and chemical denaturation in the presence and absence of AA. From the thermal denaturation, the $\mathrm{T}_{\mathrm{m}}$ and $\Delta \mathrm{G}^{\circ}{ }_{(298 \mathrm{~K})}$ magnitudes obtained were $327.7 \mathrm{~K}$ and $88 \mathrm{~kJ} / \mathrm{mol}$, respectively, for HSA alone, and $323.4 \mathrm{~K}$ and $85 \mathrm{~kJ} / \mathrm{mol}$, respectively, following treatment with a $10 \mu \mathrm{M}$ AA concentration. The same manner of reduction in Gibbs free energy as a criterion of protein stability was achieved during chemical denaturation by urea in the presence of AA. The present study investigates HSA binding nature through MD approaches, and the results indicated that the binding affinity of AA to the subdomain IIA of HSA is greater compared with that of subdomain IIIA. Although the HSA regular secondary structure evaluation by CD exhibited a minor change following incubation with AA, its tertiary structure revealed an observable fluctuation. Thus, it appears that the interaction between AA and HSA requires minor instability and partial structural changes.
\end{abstract}

\section{Introduction}

Albumin is the most abundant plasma protein (35-50 g/l human serum) with a molecular weight of $66.5 \mathrm{kDa}$. The functions

Correspondence to: Dr Nematollah Gheibi, Cellular and Molecular Research Center, Qazvin University of Medical Sciences, Shahid Bahonar Boulevard, Qazvin 3419915315, Iran

E-mail:ngheibi@qums.ac.ir

Key words: human serum albumin, arachidonic acid, structure, thermodynamic, molecular dynamics and binding properties of human serum albumin (HSA) are multifold (1). HSA facilitates the transport and disposition of various endogenous and exogenous components to their specified targets. Albumin is emerging as a versatile protein carrier for ligand targeting and also for peptide pharmacokinetic profile improvement or for protein-based ligands (2). An X-ray crystallographic study reveal that the heart shaped HSA consists of three structurally similar domains (I, II and III), each of which contains two subdomains (A and B) (3). It has been suggested that the principal regions of the ligand which bind to HSA are located in hydrophobic cavities in subdomains IIA and IIIA, which respectively are designated as sites I and II (4,5). The binding affinity offered by site I is mainly through hydrophobic interactions, whilst site II involves a combination of hydrophobic, hydrogen bonding and electrostatic interactions (6). The unique nature of the HSA ligand binding properties reflect its multi domain organization, and it is also one of the most important structure-function associations ever reported for monomeric proteins (7). HSA is known to be able to carry almost every small molecule; thus, it may be a potential contender for being a cargo molecule/or nano-vehicle for clinical, biophysical and industrial purposes $(8,9)$. This protein serves an essential function as a transporter of various unsaturated fatty acids including arachidonic acid (AA) and hormones including L-thyroxin to the target sites (10). Polyunsaturated fatty acids (PUFAs) are a group of fatty acids which contain more than one double bond in their backbone. This class comprises a number of important compounds, including essential fatty acids (11). AA is one of the most important biological lipids which is present in most organic species, is a physiologically significant omega- 6 fatty acid and also the precursor of prostaglandins and other active molecules (12). Human are able to easily metabolize linoleic acid to form n-6 PUFA. It has been suggested that AA functions as a protective agent against melanoma cancer and blood coagulation (13). Furthermore, lipid mediators generated from long-chain PUFA (AA in the $n-6$ series and eicosapentaenoic acid and docosahexaenoic acid in the n-3 series) have important functions in immune regulation and inflammation $(14,15)$. 
As mentioned above, HSA binds to a large variety of ligands and delivers them to their target organs, functioning as protein carriers. Thus, the present study aimed to identify the effects of the physiochemical properties of AA on HSA stability and structure. Their interaction was assessed based on thermodynamic, structural and molecular dynamics (MD).

\section{Materials and methods}

Materials. AA was purchased from Sigma-Aldrich; Merck KGaA. The stock solution was prepared in buffer $\left(\mathrm{KH}_{2} \mathrm{PO}_{4}=0.925 \mathrm{gr}, \mathrm{KHPO}_{4}=0.825 \mathrm{gr}, 169 \mathrm{ml}\right.$ with double-distilled water, $\mathrm{pH}=6.8$ ). HSA was purchased from Sigma-Aldrich; Merck KGaA. The HSA solutions were prepared $30 \mathrm{~min}$ prior to the experiments. In the present study, $40 \mu \mathrm{M}$ HSA and $10 \mu \mathrm{M}$ AA concentrations were used. For denaturation experiments, $6 \mathrm{M}$ urea was prepared.

HSA chemical and thermal denaturation. To evaluate the effects of AA on protein chemical stability, the HSA chemical denaturation profiles were recorded by the titration of $40 \mu \mathrm{M}$ protein solution with aliquots from a $6 \mathrm{M}$ urea stock solution. These experiments were performed in the absence and presence of a $10 \mu \mathrm{M}$ AA concentration. The protein conformational changes were obtained at wavelength of $280 \mathrm{~nm}$ by spectrophotometric technique.

Fluorescence quenching experiments. HSA thermal denaturation fluorescence measurements were performed using a spectrofluorometer (Cary Eclipse model 100, equipped with a thermostatically controlled cuvette compartment). Variable temperatures were utilized $\left(10-90^{\circ} \mathrm{C}\right)$ and the emission spectra were recorded at $357 \mathrm{~nm}$ with excitation wavelength of $290 \mathrm{~nm}$ with an increase of $1 \mathrm{~nm}$. The HSA $(40 \mu \mathrm{M})$ intrinsic fluorescence was evaluated using thermal scanning, in the absence and presence of $10 \mu \mathrm{M}$ AA.

Intrinsic fluorescence. The HSA intrinsic fluorescence evaluations were performed in the presence and absence of AA via a spectrofluorometer (Cary model Eclipse) using a $1 \mathrm{~cm}$ quartz cell and a thermostat bath. A $40 \mu \mathrm{M}$ HSA solution was prepared and titrated with increasing concentrations of AA $(0,40,80$, $120,160,200,240,280,320$ and $360 \mu \mathrm{M})$ in $50 \mathrm{mM}$ buffer solution with $298 \mathrm{~K}$ temperature. An appropriate blank which corresponded to the buffer was subtracted in order to correct for the background fluorescence. The excitation wavelength was $290 \mathrm{~nm}$, and the emission spectra were recorded from 300 to $420 \mathrm{~nm}$. The maximum emission intensities were used to calculate the binding constants, binding sites occupation and thermodynamic parameters evaluation (16). The quenching process is described by the Stern-Volmer equation: $\mathrm{F}_{0} / \mathrm{F}=1$ $+K_{\mathrm{SV}}[\mathrm{Q}]=1+k_{\mathrm{q}} \tau_{0}[\mathrm{Q}]$. Where $\mathrm{F}_{0}$ and $\mathrm{F}$ are the steady-state fluorescence intensities in the absence and in the presence of quencher (AA), respectively, $\mathrm{K}_{\mathrm{SV}}$ the Stern-Volmer quenching constant, and [Q] the concentration of the quencher. $\mathrm{kq}$ is the quenching rate constant of the biomolecule, $\tau_{0}$ is the average lifetime of the biomolecule without quencher (17).

The number of binding sites and the binding constant of the AA and HSA interaction were obtained by the following equation: $\log \left[\left(\mathrm{F}_{0}-\mathrm{F}\right) / \mathrm{F}\right]=\log \left(K_{\mathrm{b}}\right)+\mathrm{n} \log ([\mathrm{Q}])$, where $K$ is the binding constant for a site and $\mathrm{n}$ is the number of bindings per albumin, respectively. The plot of $\log \left[\left(\mathrm{F}_{0}-\mathrm{F}\right) / \mathrm{F}_{0}\right]$ versus $\log$ [Q] yields $\log \left(K_{\mathrm{b}}\right)$ as the intercept and $\mathrm{n}$ as the slope. The values for $K$ and $\mathrm{n}$ were obtained from the intercept and the slope (18). In order to map the HSA and AA interactions, the thermodynamic parameters were calculated using the Vant Hoff equation $(19,20): \ln \left(K_{\mathrm{b}}\right)=-(\Delta \mathrm{H} / \mathrm{RT})+(\Delta \mathrm{S} / \mathrm{R})$. Where the $K_{\mathrm{b}}$ is the binding constant, $\mathrm{R}$ is the universal gas constant $(8.314 \mathrm{~kJ} / \mathrm{mol}), \Delta \mathrm{H}$ and $\Delta \mathrm{S}$ are enthalpy and entropy changes during the quenching process. The free energy changes $(\Delta \mathrm{G})$ associated with the interaction of AA and HSA were calculated from the following equation: $\Delta \mathrm{G}=\Delta \mathrm{H}-\mathrm{T} \Delta \mathrm{S}=-\mathrm{RT} \ln (K)$.

Lifetime measurements. Fluorescence lifetime measurements were performed using an Edinburgh Instruments (FLS 920) spectrofluorimeter in laser mode with excitation and emission wavelengths of 255 and $320 \mathrm{~nm}$, respectively. The spectrofluorimeter in photon counting mode equipped with a temperature-controlled cell connected to a circulating water bath was used. The fluorescence lifetime of $40 \mu \mathrm{M}$ HSA in buffer phosphate $(\mathrm{pH} \mathrm{7)}$ was measured in the presence and absence of 100-fold concentrations of 0,20 and $80 \mu \mathrm{M}$ AA. Using a double-exponential decay function of the decay profiles with F900 analysis software, the lifetime values were determined from the reconvolution fit analysis. Applying the reduced $\chi^{2}$ value, the goodness of fit was assessed (close to 1 in all cases).

Furthermore, according to the following equation and using the amplitude $\beta_{i}$ with the Euler angle in the $i^{\text {th }}$ frame, component lifetime $\tau F i$, the mean fluorescence lifetime $(\tau F)$ was calculated by taking into account the amplitude $\alpha_{i}(21)$ : $\tau_{\mathrm{F}}=\Sigma_{\mathrm{i}}\left(\beta_{\mathrm{i}} \tau_{\mathrm{Fi}}^{2}\right) / \Sigma_{\mathrm{i}}\left(\alpha_{\mathrm{i}} \tau_{\mathrm{Fi}}\right)$.

Circular dichroism (CD) measurements. CD measurements were performed using a JASCO spectropolarimeter (model J-800; JASCO Applied Sciences) equipped with a thermoelectrically controlled cell holder under a constant nitrogen flow. Cuvettes with path lengths of $1 \mathrm{~mm}$ were utilized. Far-ultraviolet (UV)-CD spectra were recorded in the range of 190-260 nm. Near-UV-CD of the adjacent chain of aromatic amino acids was absorbed in the range of 250-290 nm. A concentration of $40 \mu \mathrm{M}$ HSA and AA concentrations of $0,20,80$ and $100 \mu \mathrm{M}$ were used. The slit width was set at $5 \mathrm{~nm}$, and the speed of scanning was $30 \mathrm{~nm} / \mathrm{min}^{-1}$.

Fluorescence anisotropy measurements. Fluorescence anisotropy is a phenomenon where the light emitted by a fluorophore has unequal intensities along different axes of polarization. The steady state anisotropy $(\mathrm{r})$ is given by:

$$
r=\frac{I_{V V^{-}} G x I_{V H}}{I_{V V^{+}}+G \times 2 I_{V H}}
$$

where IVV and IVH are the intensities measured with vertically polarized excitation, as indicated by the first subscript, and detected through vertically or horizontally oriented emission polarizers, respectively, as indicated by the second subscript. The factor

$$
G=\frac{I_{H V}}{I_{H H}}
$$

which is measured using horizontally polarized excitation, corrects for instrument polarization bias. Fluorescence 


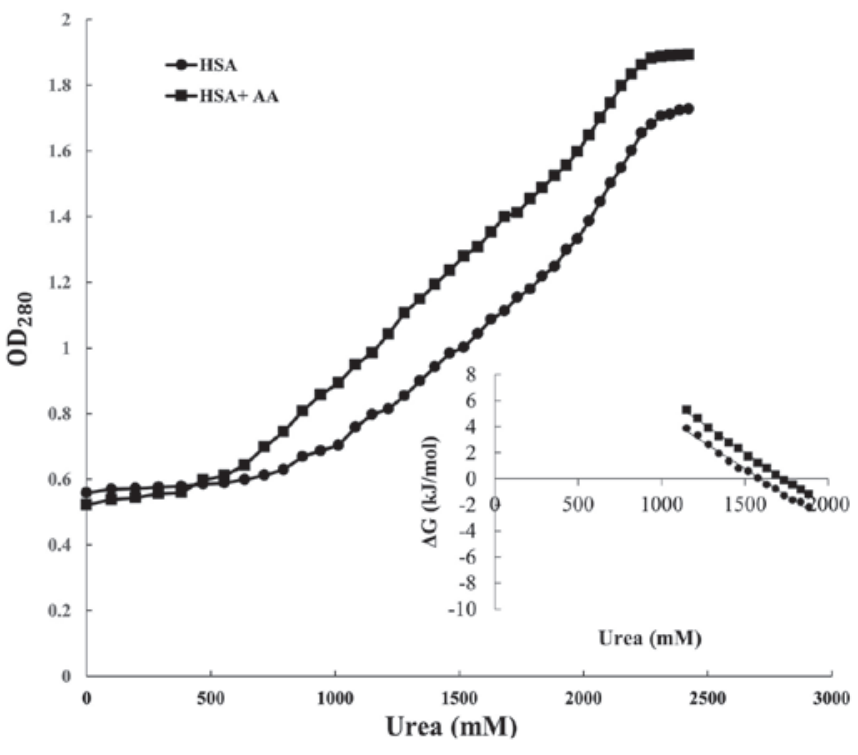

Figure 1. UV/Vis absorbance spectra of the chemical denaturation of HSA $(40 \mu \mathrm{M})$ following titration with urea, and its interaction with AA $(10 \mu \mathrm{M})$. Inset: Gibbs free energy versus urea $(\mathrm{mM})$ for the transition of HSA in the presence and absence of AA. HSA, human serum albumin; AA, arachidonic acid.

spectra and steady-state emission anisotropies of all HSA samples were measured using a Cary Eclipse fluorimeter (Agilent Technologies, Inc.). Anisotropy was measured with a manual polarizer and the $\mathrm{G}$ factor correction was performed manually, with a reference fluorophore. The labelled protein was then excited at start $(635 \mathrm{~nm})$ and stop $(640 \mathrm{~nm})$ points. Furthermore, the parallel and perpendicular emissions were monitored at maximum emission of $580 \mathrm{~nm}$ with a manual polarizer. The anisotropy measurement of the protein was then repeated with AA $(21,22)$.

Auto Dock technique (Visual MD). To study the interactions between HSA and AA, molecular docking was performed by the Auto Dock Vina 1.5.6 program). The docking process was performed to predict the preferred orientation and binding affinity of the ligands. The HSA crystal structure was obtained from a protein data bank (4L9Q PDB code). The graphical images obtained represent the best pose which were prepared using LigPlot v.4.5.3 software (23).

Protein-ligand docking. The AA molecular docking process to HSA was performed using AutoDock Vina. The docking method in which receptor side chains and the ligand are flexible was used for the aforementioned process. To assess the permissible torsions for the ligand, characterizing the search space coordinates and adding polar hydrogen atoms to the protein, the graphical AutoDock tool was applied (24). Following this, the docking process of domain I and II was performed via a grid size of $126 \times 126 \times 126$ along the X, Y and Z axes with $1 \AA$ spacing. The lowermost binding energy for the docking conformation of AA2-HSA and AA3-HSA complexes was created using AutoDock Vina, which were assumed as the primary conformation for the MD simulation process.

$M D$ of $A A$ and HSA. The conformational modifications of ligand-enzyme complexes were estimated using a MD method via the GROMACS 4.5.4 (University of Groningen Royal Institute of Technology) package software and the GROMOS96 43a1 force field. A periodic box full of water molecules was designated for the complexes which was achieved by the MD method (25). The HSA topology characteristics were created using the aforementioned GROMACS program. The AA topology parameters were created by means of the Dundee PRODRG server (26). The complex was immersed in a cubic box of extended simple point charge water molecules. The steepest descent method of 10,000 steps followed by the conjugation gradient method for 10,000 steps was utilized to minimize the energy in order to release incompatible contacts. The system equilibration phase constant substance amount, pressure and temperature (NPT) and constant substance amount, volume and temperature (NVT) as performed at $300 \mathrm{~K}$ for $200 \mathrm{psec}$ followed by MD production run for 20 nsec. As described in previous studies $(27,28)$, the atomic coordinates were registered every 2.0 psec during the MD simulation process. In this section parameters, including root mean square deviation (RMSD) and root mean square fluctuation (RMSF), were measured.

\section{Results}

Chemical and thermal denaturation. Chemical and thermal denaturation profiles were obtained from urea titration and thermal scanning in the absence and presence of AA in specific concentrations, which are depicted in Figs. 1 and 2. Each profile is a sigmoidal curve; thus, this process is described as a single denaturant-dependent step based on the two-step theory. Determination of the standard Gibbs free energy of denaturation $\left(\Delta \mathrm{G}^{0}\right)$, as a criterion of the conformational stability of a globular protein, is based on two state theory and the equation 1 (29): $\Delta \mathrm{G}^{0}=-\mathrm{RT} \ln (\mathrm{K})$. The secondary plots of Figs. 1 and 2 are illustrated insets. From these linear plots, the $\Delta \mathrm{G}^{0}$ varies linearly in a denaturation-dependent manner (urea concentration and temperature) over a limited region. The equation for determining the $\Delta \mathrm{G}^{0}$ is as follows: $\Delta \mathrm{G}^{0}=\Delta \mathrm{G}^{0}\left(\mathrm{H}_{2} \mathrm{O}\right)-\mathrm{m}$ [denaturant]. Where $\Delta \mathrm{G}^{0}\left(\mathrm{H}_{2} \mathrm{O}\right)$ is the free energy of conformational stability in the absence of a denaturant, while $\mathrm{m}$ is the measure of $\Delta \mathrm{G}^{0}$ dependence in denaturant concentration.

In chemical denaturation, [Urea $]_{1 / 2}$ is the denaturant concentration which the protein needs to reach to halve its two-state transition. In thermal denaturation, the protein melting point $\left(\mathrm{T}_{\mathrm{m}}\right)$ is a temperature which the protein needs to reach to halve its two-state transition. The magnitudes of $\Delta \mathrm{G}^{0}\left(\mathrm{H}_{2} \mathrm{O}\right)$, [Urea $]_{1 / 2}$ and $\mathrm{T}_{\mathrm{m}}$ were determined from replots, and are summarized in Table I.

Internal fluorescence experiments. Internal fluorescence spectroscopy is a method which is widely used to investigate the microenvironment of amino acid residues by measuring the emission wavelength shift (30). The HSA internal fluorescence spectra with various amounts of AA are presented in Fig. 3. The HSA fluorescence intensity regularly decreased with the addition of AA. Furthermore, it was demonstrated that fluorescence quenching occurred in the binding. Additionally, it implies that the interaction between AA and HSA may affect the Trp residue microenvironment. Thermodynamic parameters of the binding reaction, obtained from the Stern-Volmer curve, confirm the 
Table I. Thermodynamic parameters obtained from the chemical and thermal denaturation curves.

\begin{tabular}{lccccccc}
\hline & \multicolumn{3}{c}{ Chemical denaturation } & & \multicolumn{2}{c}{ Thermal denaturation } \\
\cline { 2 - 3 } Sample & {$[$ Ligand $] / 2, \mathrm{M}$} & $\Delta \mathrm{G}\left(\mathrm{H}_{2} \mathrm{O}\right), \mathrm{kJ} / \mathrm{mol}$ & & $\mathrm{M}, \mathrm{kJ} / \mathrm{mol}$ & & $\mathrm{T}_{\mathrm{m}}, \mathrm{K}$ & $\Delta \mathrm{G}^{0}(298 \mathrm{~K}), \mathrm{kJ} / \mathrm{mol}$ \\
\hline HSA & 0.16 & 13.19 & 83 & & 327.7 & 88 \\
HSA/AA & 0.13 & 9.6 & 72 & & 323.4 & 85
\end{tabular}

HSA, human serum albumin; AA, arachidonic acid; M, Line slope.

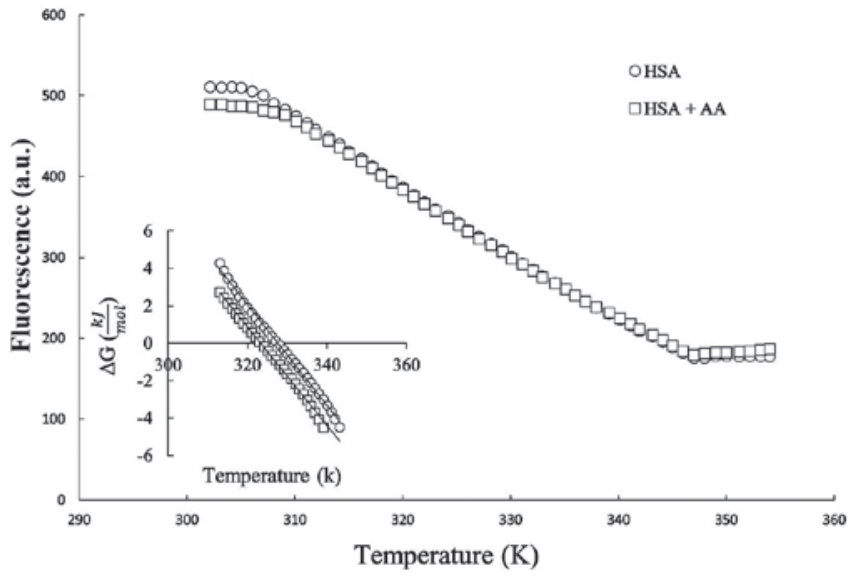

Figure 2. Thermal denaturation curve obtained from emission internal fluorescence of HSA $(40 \mu \mathrm{M})$ and its interaction with AA $(10 \mu \mathrm{M})$. Inset: Effects of AA on fluorescence spectrum of HSA. HSA, human serum albumin; AA, arachidonic acid.

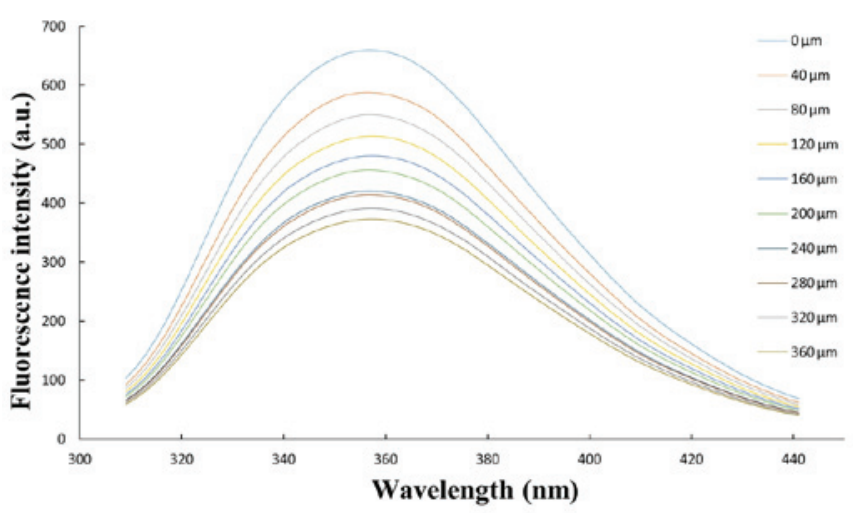

Figure 3. Curve obtained from fluorescence spectrum of HSA ( $T=300 \mathrm{~K}$, excitation wavelength, $290 \mathrm{~nm}$ ) in the presence of a range of concentrations of arachidonic acid $(0,40,80,120,160,200,240,280,320$ and $360 \mu \mathrm{M})$, with $\mathrm{HSA}=40 \mu \mathrm{M}$. HSA, human serum albumin.

binding force. Therefore, the temperature-dependent thermodynamic parameters were analyzed to characterize the acting forces between AA and HSA (31). The results of the data sequential analysis using the Vant Hoff equation are illustrated in Table II. These were confirmed parameters by Fig. 4.

Lifetime measurements. Based on the results of the fluorescence lifetime measurements, adding 20 and $80 \mu \mathrm{M}$ AA to the protein solution resulted in no significant change in the

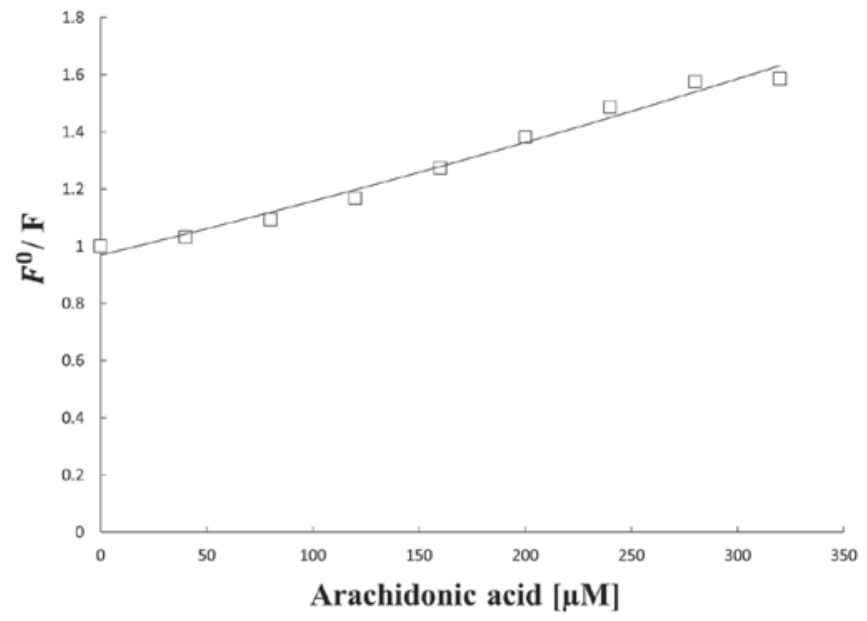

Figure 4. Stern-Volmer curve for the interaction between human serum albumin and arachidonic acid.

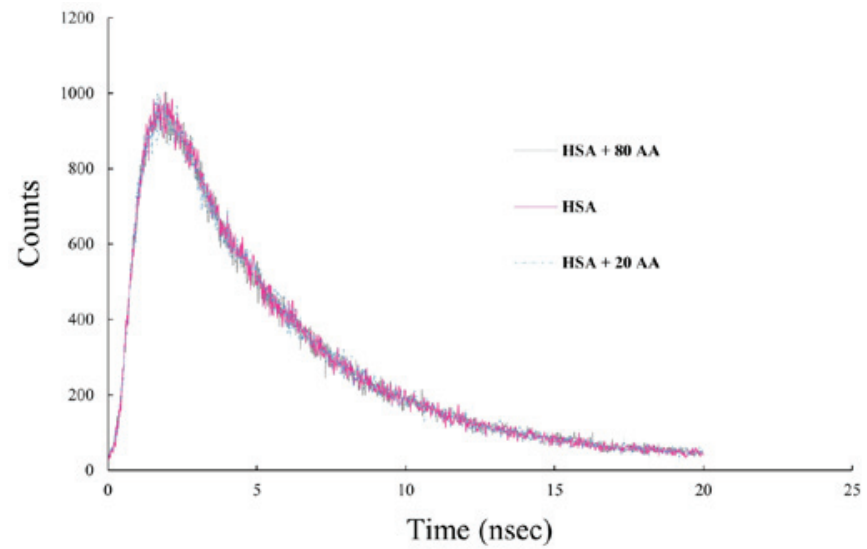

Figure 5. Lifetime measurements of the HSA/AA interaction: $\mathrm{HSA}=40 \mu \mathrm{M}$ and $\mathrm{AA}=20$ and $80 \mu \mathrm{m}$. HSA, human serum albumin; AA, arachidonic acid.

fluorescence lifetime of HSA (Fig. 5). Therefore, changing the concentration AA over time does not change, which indicates that the interaction is static.

$C D$ spectroscopic experiments. The far-UV-CD spectra of HSA exhibited two negative bands in the $208 \mathrm{~nm}$ and $222 \mathrm{~nm}$ regions, which represent the $\beta$-sheet and $\alpha$-helical structure of the protein, respectively. The near-UV-CD method obtained the HSA tertiary regular structure values (32). When the AA concentration was gradually increased from 20 to $100 \mu \mathrm{M}$, the $\mathrm{CD}$ exhibited fundamental changes (Fig. 6A and B). 
Table II. Binding and thermodynamic parameters obtained from the Stern-Volmer equation following the interaction of HSA/AA.

\begin{tabular}{lccccccc}
\hline Sample & $\mathrm{T}, \mathrm{K}$ & $\mathrm{n}$ & $\mathrm{R}$ & $\mathrm{K}_{\mathrm{SV}}, 10^{4} \mathrm{l} / \mathrm{mol}$ & $\Delta \mathrm{G}^{0}, \mathrm{~kJ} / \mathrm{mol}$ & $\Delta \mathrm{H}^{0}, \mathrm{~kJ} / \mathrm{mol}$ & $\Delta \mathrm{S}^{0}, \mathrm{~J} /(\mathrm{mol} \mathrm{K})$ \\
\hline HSA/AA & 298 & 2 & 0.98 & 5.3 & -31.13 & -22.66 & 28.42
\end{tabular}

HSA, human serum albumin; AA, arachidonic acid. R, regression analysis is a set of statistical processes for estimating the relationships.
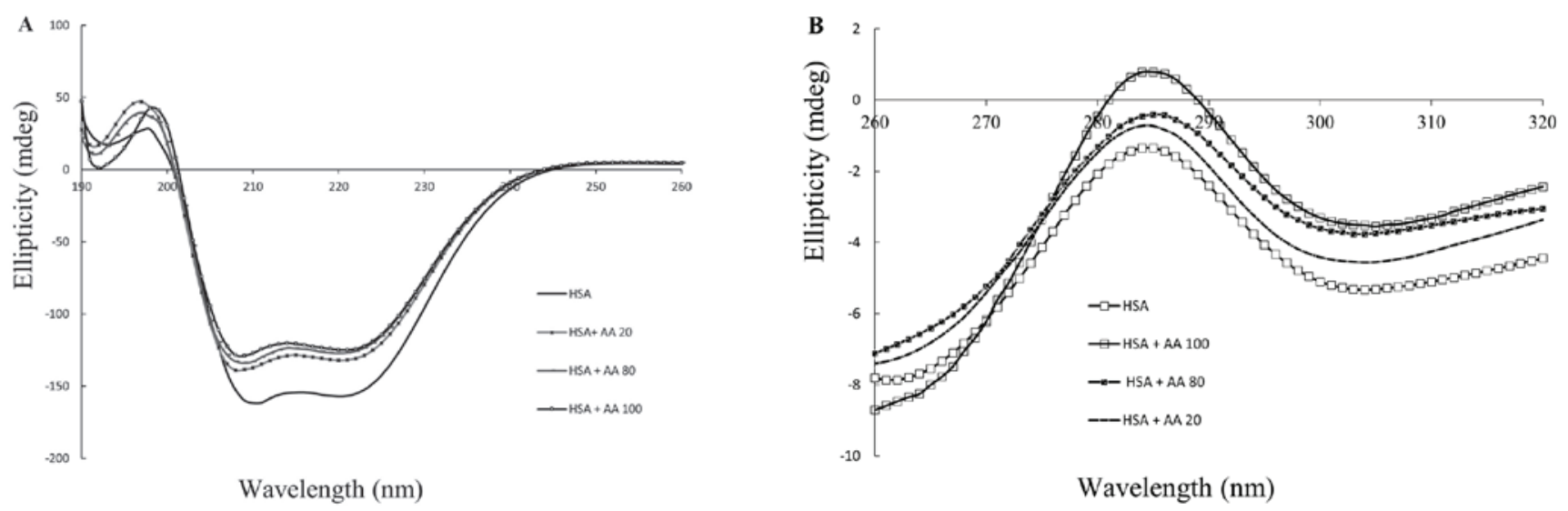

Wavelength (nm)

Figure 6. UV-CD spectroscopic experiments of the interaction between HSA and AA. (A) Far-UV-CD spectra of the HSA/AA interaction. (B) Near-UV-CD spectra of the HSA/AA interaction; with $\mathrm{HSA}=40 \mu \mathrm{M}$ and $\mathrm{AA}=20,80$ and $100 \mu \mathrm{m}(\mathrm{B})$. HSA, human serum albumin; $\mathrm{AA}$, arachidonic acid; CD, circular dichroism.

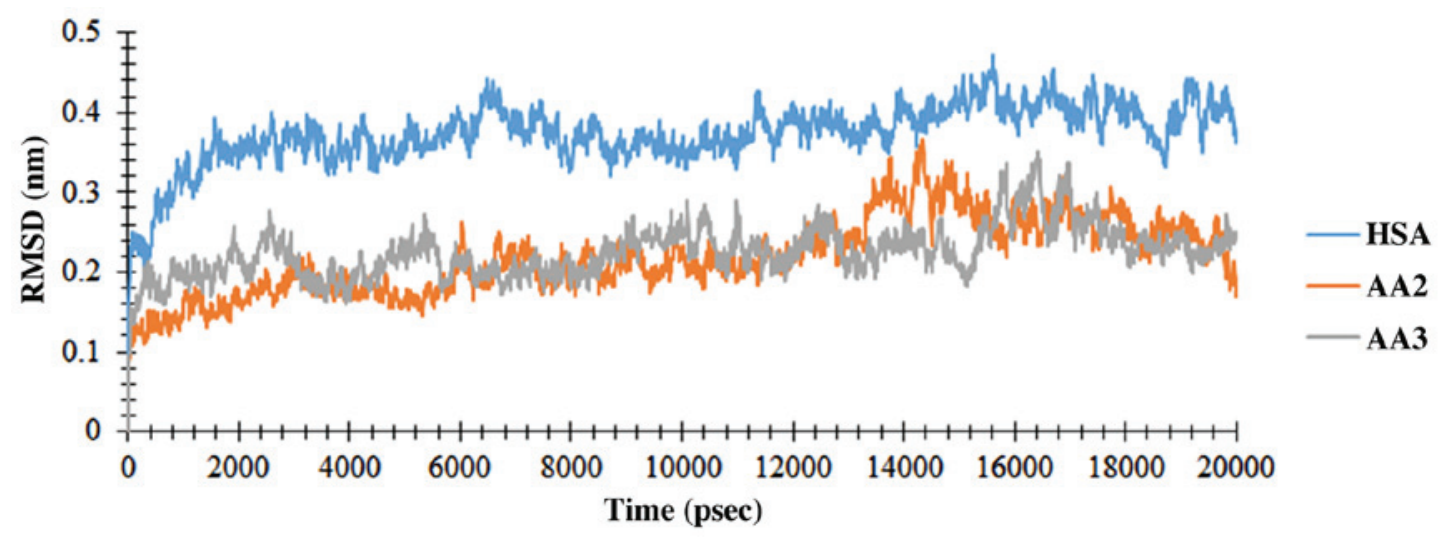

Figure 7. RMSD values of carbon- $\alpha$ atoms of free HSA and HSA in complex with AA. Free HSA (blue), AA2 (orange) and AA3 (grey). RMSD, root means square deviations; HSA, human serum albumin; AA, arachidonic acid.

Anisotropy measurement. HSA was labeled with a fluorophore and the anisotropy of the labeled protein was measured prior to and following AA binding. The dye to protein ratio was low and the anisotropy of unlabeled protein was 0.28 . When AA was added to the labeled protein, the anisotropy decreased to 0.26. Interactions between HSA and AA and the third structure are not stable. This may be attributed to conformational changes in HSA and the generation of another binding site upon interaction with AA, which are in agreement with our $\mathrm{CD}$ and anisotropy results (33).

Molecular docking and dynamics. To determine the interaction of AA with HSA specific binding sites, a molecular docking process was performed. The main result to note of the reaction is the binding position of AA with HSA. One previous study demonstrated which knowledge about the protein-ligand binding may be useful to understand the function and effectiveness of AA and HSA as potential therapeutic agents (34). The selective side-chain residue flexibility is a great option which is available on AutoDock Vina docking software (35). The aim of the present study was to make the protein-ligand interaction environment more pragmatic in order to decrease the computer processing time. The conformational modification of the receptor with regards to ligand selectivity demonstrate that receptor flexibility may have an essential function in computer drug design, which is evaluated in current study (36). To determine the structural changes, which are induced by ligand binding (with AA), the MD simulation for free HSA 

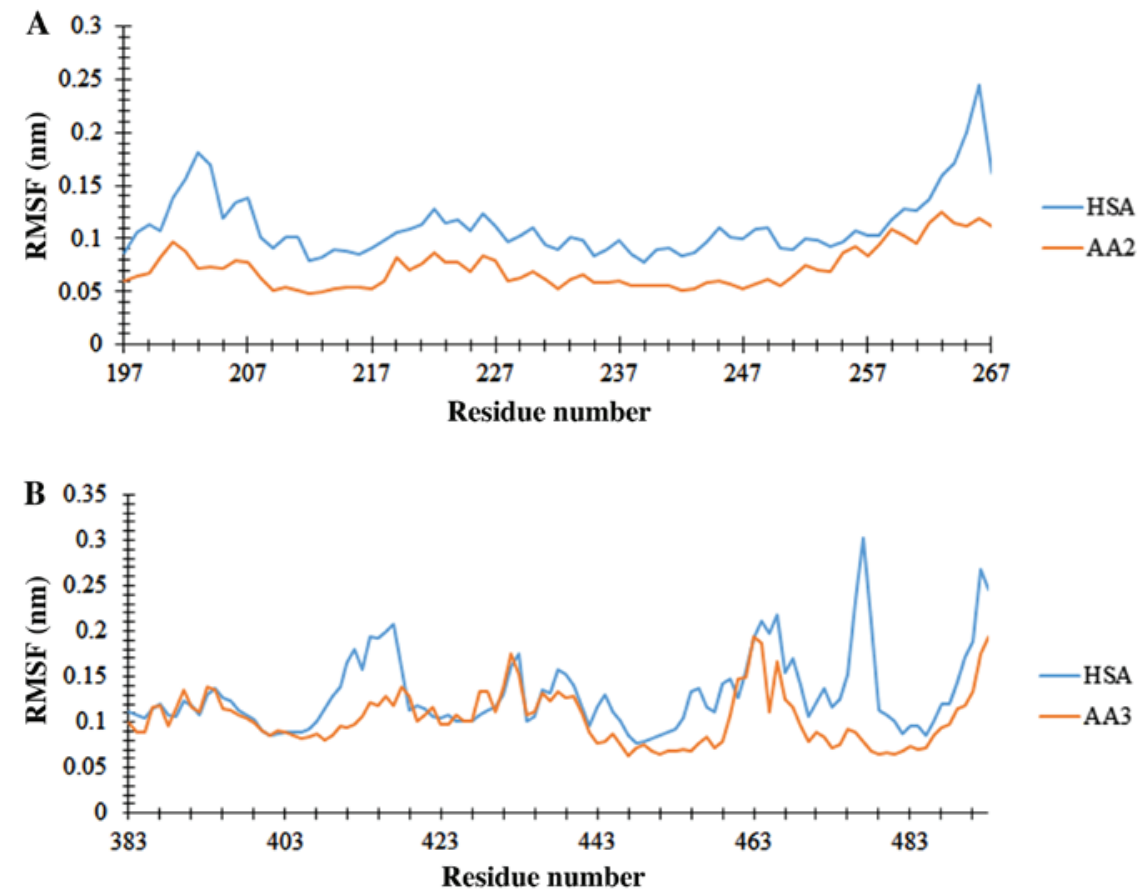

Figure 8. RMSF of free HSA and HSA in complex with AA. (A) In subdomain IIA, free HSA (blue) and AA2-HSA (orange); (B) in subdomain IIIA, free HSA (blue) and AA3-HSA (orange). RMSF, root mean square fluctuation; HSA, human serum albumin; AA, arachidonic acid.
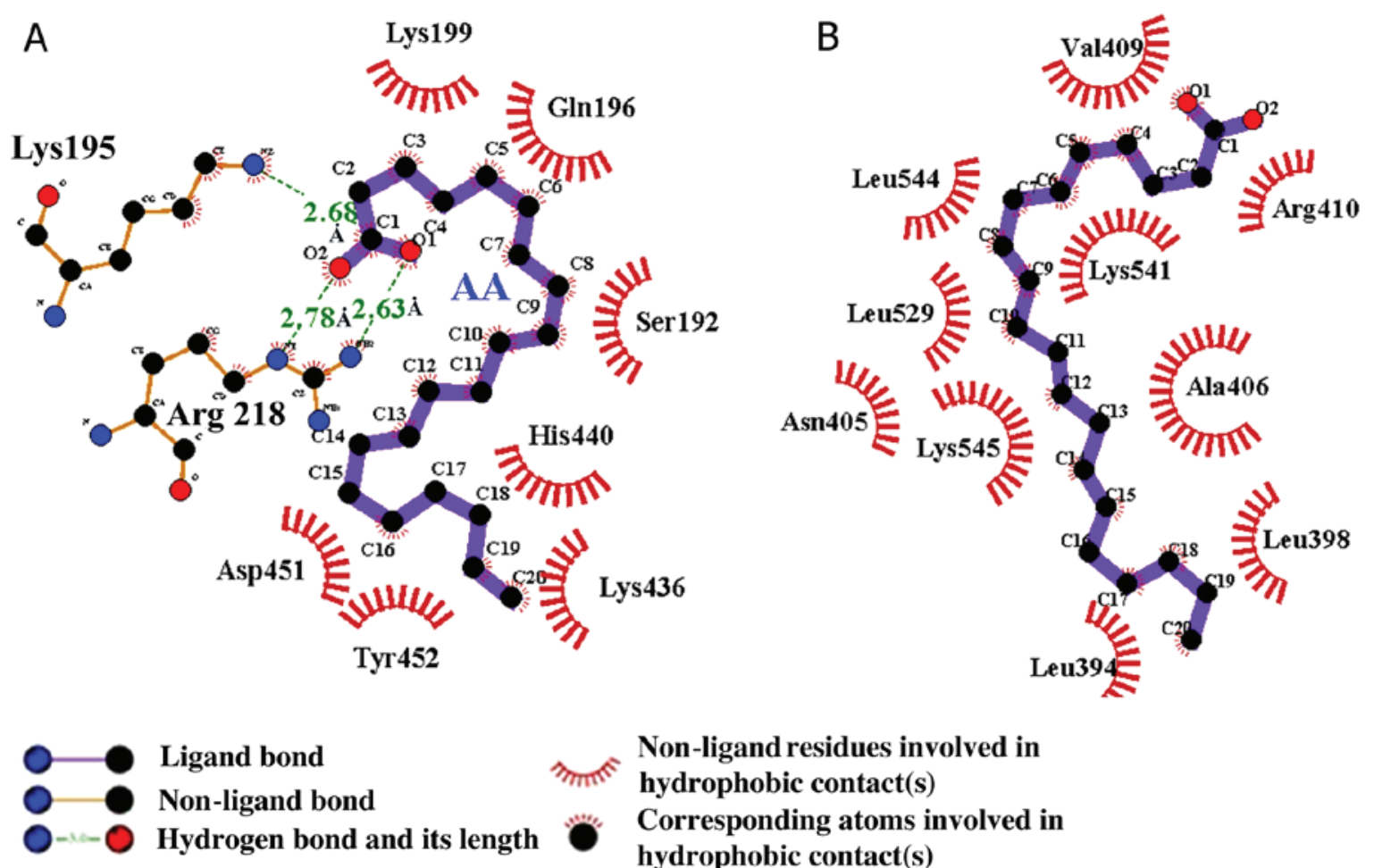

\section{Ligand bond}

Non-ligand bond

Hydrogen bond and its length
Non-ligand residues involved in

hydrophobic contact(s)

Corresponding atoms involved in

hydrophobic contact(s)

Figure 9. Best docked conformations of (A) AA2-HSA and (B) AA3-HSA. HSA, human serum albumin; AA, arachidonic acid.

(subdomain II and III) and AA2-HSA/AA3-HSA complexes was performed and the results were consequently compared. Based on structural characteristics, the stability of the HSA protein was able to be studied during the simulation time. The evolution time of RMSD from the preliminary structure was calculated for three simulation runs. The RMSDs of $\mathrm{C} \alpha$ atoms, which are representative of two complexes, are demonstrated in Fig. 7. The free protein and the protein involved in the complex achieved the appropriate stability after 1 psec, which proved the equilibration of the system. The RMSD values of the HSA $\mathrm{C} \alpha$-atoms and the complexes indicated a near mean equilibration and oscillation value for the whole system with maintained stability up to the end of the simulation. To obtain the flexibility data, RMSF was measured. 
To identify the flexible regions of the molecule, RMSFs of $\mathrm{C} \alpha$ atoms were produced and are illustrated in Fig. 8. The RMSF value for the free protein and the protein involved in the complex have similar trends as illustrated in Fig. 8, while residues 262-267 and 474-480 in subdomain IIA and IIIA have the highest RMSF values. Due to molecular movement restriction which was induced by the ligand, the free protein demonstrates a higher RMSF value compared with the protein involved in the complex. Following MD analysis, the AA-HSA complex in site I was formed by the hydrogen bonds of Lys195 and Arg218 and hydrophobic interactions, as indicated in Fig. 9A. Additionally, the AA-HSA complex was created by the hydrophobic interaction in site II, as illustrated in Fig. 9B. The optimal probable conformation was obtained with AA and HSA following docking and MD analysis. The molecular affinity of AA2 and AA3 with HSA was reported as -5.8 and $-5.0 \mathrm{kcal} / \mathrm{mol}$, respectively. These results demonstrated which AA binds forcefully to the large hydrophobic cavity available on HSA at subdomain IIA of site I. Studies have confirmed that IIA and IIIA subdomains are suitable as target and initial drug binding sites for the docking process. Each experiment which evaluates the ligand-binding site next to drug-binding sites I/II may be useful in selecting the best docking simulation position. To measure the structural modifications caused by the ligand (AA) binding the MD simulation process for free HSA and IIA/IIIA subdomains, experiments were performed and the results consequently compared. At present, the results demonstrated that AA has the ability to bind effectively to the large hydrophobic cavity, which is available on HSA at subdomain IIA of site I. The HSA binding site was evaluated to recognize the site containing residues.

\section{Discussion}

A large number of previous studies indicated that IIA/IIIA subdomains may be regarded as target sites for docking due to their early drug binding site potential. Appraising other binding sites may be more useful for a suitable site selection for the MD simulation process. To evaluate the structural modifications, which are provided by ligand (AA) binding, in the present study, the MD simulation for HSA and IIA/IIIA subdomains was performed and the results compared $(37,38)$. Thermodynamic parameters, which were obtained from the chemical and thermal denaturation plots of HSA and AA, revealed that the protein Gibbs free energy and $\mathrm{T}_{\mathrm{m}}$ decreased following incubation with AA. This instability was proven by structural analysis from fluorescence and CD studies. The effects of AA binding on HSA stability was investigated by urea-induced denaturation using optical density 280 measurements via spectrophotometry and its thermal denaturation following protein excitation in $290 \mathrm{~nm}$ wavelength via spectrofluorometer techniques (Figs. 1 and 2). The Gibbs free energy of $\operatorname{HSA}\left(\Delta \mathrm{G}_{\mathrm{H}_{2} \mathrm{O}}\right)$ in the absence and present of AA was revealed to be 13.19 and $9.6 \mathrm{~kJ} / \mathrm{mol}$, respectively, as determined by the linear fittings of intrinsic spectrophotometric data. As presented in Table I, all the thermodynamic parameters including $\Delta \mathrm{G}_{\mathrm{H}_{2} \mathrm{O}}, \mathrm{C}_{\mathrm{m}}$, $\Delta \mathrm{G}_{298 \mathrm{~K}}$ and $\mathrm{T}_{\mathrm{m}}$ which are achieved from chemical and thermal denaturation, revealed a relative HSA instability following its interaction with AA (39). In the chemical denaturation, the urea interacts with HSA by electrostatic forces, yielding a randomly coiled conformation in its unfolded state, while the thermal denaturation produces a molten globule state and the protein aggregation; therefore, each method yield different structurally unfolded states of HSA. From the efonidipine interaction with BSA, it may be seen that $\Delta \mathrm{H}=68.04 \mathrm{~kJ} / \mathrm{mol}$, $\Delta \mathrm{S}=319.42 \mathrm{~kJ} / \mathrm{mol}$ and $\Delta \mathrm{G}=-27.08 \mathrm{~kJ} / \mathrm{mol}(40)$. These positive values of $\Delta \mathrm{H}$ and $\Delta \mathrm{S}$ are associated with the hydrophobic nature of the ligand-protein interaction. The negative value of $\Delta \mathrm{G}^{0}$, which is obtained from Stern-Volmer data analysis as presented in Table II, identifies that the HSA-AA binding is a spontaneous process. The positive $\Delta \mathrm{H}^{0}$ and $\Delta \mathrm{S}^{0}$ values for AA indicate that the force acting between these compound and AA is mainly a hydrophobic interaction. Thus, the HSA non-polar hydrophobic groups may be responsible for the main effect determination of AA-protein binding (41). As demonstrated in Table II, the $K_{\mathrm{SV}}$ values may be obtained from the modified linear Stern-Volmer equation slope. Additionally, the $K_{\mathrm{SV}}$ of the AA interaction with HSA suggests that the overall quenching is dominated by static quenching $(42,43)$. This additionally reflects the lifetime measurements, in that the interaction between HSA and AA is of a static nature. Hence, AA may strongly quench the intrinsic fluorescence of HSA by static quenching (44). HSA structural experiments, which were evaluated by CD spectra in absence and presence of B12 and 4-thio-5-methyluridine, exhibited two negative bands in the UV region at 209 and $228 \mathrm{~nm}$, which are the protein characteristics of a $\beta$-sheet and a-helical structures. The results revealed that B12 and 4-thio-5-methyluridine had fundamental effects on HSA secondary structure $(45,46)$, and the results are in agreement with the results of the present study obtained by CD from a HSA regular secondary structure subsequent to its interaction with different concentrations of AA (Fig. 6A). The near-UV-CD results of the aforementioned previous study are consistent with our data which was provided by $\mathrm{CD}$ from a regular tertiary structure of HSA following its interaction with different concentrations of AA (Fig. 6B). The docking and MD results indicated the effective binding of AA to IIA/IIIA subdomains (site I and II) in HSA.

To conclude, based on the structure-function association experiments and the function of HSA as a carrier protein, it is necessary to change the structure and accept AA as a ligand. These changes may be explained by the nature of non-covalent physical interactions, which are induced by the ligand minor instability and flexibility.

\section{Acknowledgements}

Not applicable.

\section{Funding}

The authors acknowledged the facilitative support of the educational department of Azad University of Science and Research, the Qazvin University of Medical Sciences and the Biochemistry-Biophysics Institute of Tehran University.

\section{Availability of data and materials}

All the datasets generated and analyzed during the present study are included in the published manuscript. 


\section{Authors' contributions}

FMV drafted the manuscript, and analyzed and interpreted the data. AF and HS conducted the study and analyses, and collected background information. NG participated in the design of the present study and performed the statistical analysis. All authors read and approved the final version of the manuscript.

\section{Ethics approval and consent to participate}

Not applicable.

\section{Patient consent for publication}

Not applicable.

\section{Competing interests}

The authors declare that they have no competing interests.

\section{References}

1. Kratz F: Albumin as a drug carrier: Design of prodrugs, drug conjugates and nanoparticles. J Control Release 132: 171-183, 2008.

2. Sarkar D, Mahata A, Das P, Girigoswami A, Ghosh D and Chattopadhyay N: Deciphering the perturbation of serum albumins by a ketocyanine dye: A spectroscopic approach J Photochem Photobiol B 96: 136-143, 2009.

3. Chatterjee T, Pal A, Dey S, Chatterjee BK and Chakrabarti P: Interaction of virstatin with human serum albumin: Spectroscopic analysis and molecular modeling. PLoS One 7: e37468, 2012.

4. Elsadek B and Kratz F: Impact of albumin on drug delivery--new applications on the horizon. J Control Release 157: 4-28, 2012.

5. Lhiaubet-Vallet V, Sarabia Z, Boscá F and Miranda MA: Human serum albumin-mediated stereodifferentiation in the triplet state behavior of (S)- and (R)-carprofen. J Am Chem Soc 126: 9538-9539, 2004.

6. Jiménez MC, Miranda MA and Vayá I: Triplet excited States as chiral reporters for the binding of drugs to transport proteins. J Am Chem Soc 127: 10134-10135, 2005.

7. Fasano M, Curry S, Terreno E, Galliano M, Fanali G, Narciso P, Notari S and Ascenzi P: The extraordinary ligand binding properties of human serum albumin. IUBMB Life 57: 787-796, 2005.

8. Ahmed-Ouameur A, Diamantoglou S, Sedaghat-Herati MR Nafisi Sh, Carpentier R and Tajmir-Riahi HA: The effects of drug complexation on the stability and conformation of human serum albumin: Protein unfolding. Cell Biochem Biophys 45: 203-213, 2006.

9. Varshney A, Sen P, Ahmad E, Rehan M, Subbarao N and Khan RH: Ligand binding strategies of human serum albumin: How can the cargo be utilized? Chirality 22: 77-87, 2010.

10. Anand U and Mukherjee S: Binding, unfolding and refolding dynamics of serum albumins. Biochim Biophys Acta 1830: 5394-5404, 2013.

11. Petitpas I, Petersen CE, Ha C-E, Bhattacharya AA, Zunszain PA, Ghuman J, Bhagavan NV and Curry S: Structural basis of albumin-thyroxine interactions and familial dysalbuminemic hyperthyroxinemia. Proc Natl Acad Sci USA 100: 6440-6445, 2003.

12. Zajdel A, Wilczok A, Chodurek E, Gruchlik A and Dzierzewicz Z Polyunsaturated fatty acids inhibit melanoma cell growth in vitro. Acta Pol Pharm 70: 365-369, 2013.

13. Nelson GJ, Schmidt PC, Bartolini G, Kelley DS and Kyle D: The effect of dietary arachidonic acid on platelet function, platelet fatty acid composition, and blood coagulation in humans Lipids 32: 421-425, 1997.

14. Zhang C, Li A, Gao S, Zhang X and Xiao H: The TIP30 protein complex, arachidonic acid and coenzyme A are required for vesicle membrane fusion. PLoS One 6: e21233, 2011.

15. Høstmark AT and Haug A: Percentage oleic acid is inversely related to percentage arachidonic acid in total lipids of rat serum. Lipids Health Dis 12: 40, 2013.
16. Guo X-J, Sun X-D and Xu S-K: Spectroscopic investigation of the interaction between riboflavin and bovine serum albumin. $\mathrm{J}$ Mol Struct 931: 55-59, 2009.

17. Gao H, Lei L, Liu J, Kong Q, Chen X and Hu Z: The study on the interaction between human serum albumin and a new reagent with antitumour activity by spectrophotometric methods. J Photochem Photobiol Chem 167: 213-221, 2004.

18. Ahmad E, Sen P and Khan RH: Structural stability as a probe for molecular evolution of homologous albumins studied by spectroscopy and bioinformatics. Cell Biochem Biophys 61: 313-325, 2011.

19. Ghosh KS, Sahoo BK and Dasgupta S: Spectrophotometric studies on the interaction between (-)-epigallocatechin gallate and lysozyme. Chem Phys Lett 452: 193-197, 2008.

20. Ross PD and Subramanian S: Thermodynamics of protein association reactions: Forces contributing to stability. Biochemistry 20: 3096-3102, 1981.

21. Nigen M, Le Tilly V, Croguennec T, Drouin-Kucma D and Bouhallab S: Molecular interaction between apo or holo $\alpha$-lactalbumin and lysozyme: Formation of heterodimers as assessed by fluorescence measurements. Biochim Biophys Acta 1794: 709-715, 2009.

22. Rusinova E, Tretyachenko-Ladokhina V, Vele OE, Senear DF and Alexander Ross JB: Alexa and Oregon Green dyes as fluorescence anisotropy probes for measuring protein-protein and protein-nucleic acid interactions. Anal Biochem 308: 18-25, 2002.

23. Laskowski RA and Swindells MB: LigPlot+: multiple ligandprotein interaction diagrams for drug discovery. J Chem Inf Model 51: 2778-2786, 2011.

24. Trott $\mathrm{O}$ and Olson AJ: AutoDock Vina: Improving the speed and accuracy of docking with a new scoring function, efficient optimization, and multithreading. J Comput Chem 31: 455-461, 2010.

25. Morris GM, Goodsell DS, Halliday RS, Huey R, Hart WE, Belew RK and Olson AJ: Automated docking using a Lamarckian genetic algorithm and an empirical binding free energy function. J Comput Chem 19: 1639-1662, 1998.

26. Lindahl E, Hess B and Van Der Spoel D: GROMACS 3.0: A package for molecular simulation and trajectory analysis. Mol Model Annu 7: 306-317, 2001.

27. Farasat A, Rahbarizadeh F, Hosseinzadeh G, Sajjadi S, Kamali M and Keihan AH: Affinity enhancement of nanobody binding to EGFR: In silico site-directed mutagenesis and molecular dynamics simulation approaches. J Biomol Struct Dyn 35: $1710-1728,2017$.

28. Hansson T, Oostenbrink C and van Gunsteren W: Molecular dynamics simulations. Curr Opin Struct Biol 12: 190-196, 2002.

29. Schmittschmitt JP and Scholtz JM: The role of protein stability, solubility, and net charge in amyloid fibril formation. Protein Sci 12: 2374-2378, 2003.

30. Ashoka S, Seetharamappa J, Kandagal P and Shaikh S: Investigation of the interaction between trazodone hydrochloride and bovine serum albumin. J Lumin 121: 179-186, 2006.

31. Vekshin IL: Separation of the tyrosine and tryptophan components of fluorescence using synchronous scanning method. Biofizika 41: 1176-1179, 1996 (In Russian).

32. Gokara M, Sudhamalla B, Amooru DG and Subramanyam R: Molecular interaction studies of trimethoxy flavone with human serum albumin. PLoS One 5: e8834, 2010.

33. Zhong D, Douhal A and Zewail AH: Femtosecond studies of protein-ligand hydrophobic binding and dynamics: Human serum albumin. Proc Natl Acad Sci USA 97: 14056-14061, 2000.

34. Gokara M, Narayana VV, Sadarangani V, Chowdhury SR, Varkala S, Ramachary DB and Subramanyam R: Unravelling the binding mechanism and protein stability of human serum albumin while interacting with nefopam analogues: A biophysical and insilico approach. J Biomol Struct Dyn 35: 2280-2292, 2017.

35. Abreu RM, Froufe HJ, Queiroz MJR and Ferreira IC: Selective flexibility of side-chain residues improves VEGFR-2 docking score using AutoDock Vina. Chem Biol Drug Des 79: 530-534, 2012.

36. Mohan V, Gibbs AC, Cummings MD, JaegerEP and DesJarlais RL: Docking: Successes and challenges. Curr Pharm Des 11: 323-333, 2005.

37. Gou Y, Zhang Z, Li D, Zhao L, Cai M, Sun Z, Li Y, Zhang Y, Khan $\mathrm{H}$, Sun $\mathrm{H}$, et al: HSA-based multi-target combination therapy: Regulating drugs' release from HSA and overcoming single drug resistance in a breast cancer model. Drug Deliv 25: 321-329, 2018.

38. Shahabadi N, Fili SM and Kashanian S: Human serum albumin interaction studies of a new copper (II) complex containing ceftobiprole drug using molecular modeling and multispectroscopic methods. J Coord Chem 71: 329-341, 2018. 
39. Shahabadi N, Bazvandi B and Taherpour A: Synthesis, structural determination and HSA interaction studies of a new water-soluble $\mathrm{Cu}$ (II) complex derived from 1,10-phenanthroline and ranitidine drug. J Coord Chem 70: 3186-3198, 2017.

40. Wang N, Ye L, Zhao BQ and Yu JX: Spectroscopic studies on the interaction of efonidipine with bovine serum albumin. Braz $J$ Med Biol Res 41: 589-595, 2008.

41. Lori C, Lantella A, Pasquo A, Alexander LT, Knapp S, Chiaraluce $\mathrm{R}$ and Consalvi V: Effect of single amino acid substitution observed in cancer on Pim-1 kinase thermodynamic stability and structure. PLoS One 8: e64824, 2013.

42. Yuan T, Weljie AM and Vogel HJ: Tryptophan fluorescence quenching by methionine and selenomethionine residues of calmodulin: Orientation of peptide and protein binding. Biochemistry 37: 3187-3195, 1998.

43. Li S, Huang K, Zhong M, Guo J, Wang WZ and Zhu R: Comparative studies on the interaction of caffeic acid, chlorogenic acid and ferulic acid with bovine serum albumin. Spectrochim Acta A Mol Biomol Spectrosc 77: 680-686, 2010.
44. OAbou-Zied OK and Al-Shihi OI: Characterization of Subdomain IIA Binding Site of Human Serum Albumin in its Native, Unfolded, and Refolded States Using Small Molecular Probes. J Am Chem Soc 130: 10793-10801, 2008.

45. Hou HN, Qi ZD, Ouyang YW, Liao FL, Zhang Y and Liu Y: Studies on interaction between Vitamin B12 and human serum albumin. J Pharm Biomed Anal 47: 134-139, 2008.

46. Zhang HM, Chen TT, Zhou QH and Wang YQ: Binding of caffeine, theophylline, and theobromine with human serum albumin: A spectroscopic study. J Mol Struct 938: 221-228, 2009.

This work is licensed under a Creative Commons Attribution-NonCommercial-NoDerivatives 4.0 International (CC BY-NC-ND 4.0) License. 